\title{
Evaluation of Retinal Pigment Epithelial Hamartoma Using Oct - A Case Summary
}

\author{
P. Sukanya ${ }^{1}$, M. Prathiba ${ }^{1}$, S. Saravanan ${ }^{1}$, C.G. Vanila ${ }^{1}$, J. Kaliamurthy ${ }^{1 *}$ \\ ${ }^{1}$ Institute of Ophthalmology, Joseph Eye Hospital, Tiruchirappalli - 620 001, Tamil Nadu, India
}

Received: 19 November, 2016; Accepted: 01 December, 2016; Published: 12 December, 2016

*Corresponding author: J. Kaliamurthy, Institute of Ophthalmology, Joseph Eye Hospital, Tiruchirappalli, Tamil Nadu, India, Tel: +91-431-2460622, Mob: +91-9894540875; E-mail: kaliaj17@gmail.com

\begin{abstract}
We report a case of healthy sixteen year old girl who presented with left eye defective vision with a Snellen best corrected visual acuity of 6/36. Although the anterior segment was normal, a black retinal lesion at the fovea was detected by fundus examination. The right eye had $6 / 6$ vision and fundus examination was normal.

In the left eye the fundus fluorescein angiography features and spectral domain optical coherence tomography findings were compatible with a congenital simple hamartoma of retinal pigment epithelium (CSHRPE) at fovea. It is essential to detect this tumour and differentiate it from other pigmented retinal lesions.
\end{abstract}

\section{Introduction}

Tumours of the Retinal Pigment Epithelium (RPE) are uncommon. They are classified into four groups including CSHRPE, congenital hypertrophy of the RPE, combined hamartoma of the retina and RPE and adenoma or adenocarcinoma of the RPE [1-2].

Congenital simple hamartoma of the RPE was first recognized by Laqua [3] in 1981. Gass [1] coined the term retinal hamartoma in 1989. Since then, very few cases are reported in the literature [3-4].

The largest case series has been reported by Shields et al. in 2003 and included 5 cases [5]. We report ophthalmoscopic and imaging findings (FFA, OCT, B Scan) of a case with CSHRPE at fovea.

\section{Case Report}

A healthy 16 year old girl presented with defective vision in the left eye - Snellen BCVA was 6/36 not improving with pinhole and $6 / 6$ in the right eye. Slit-lamp biomicroscopic findings and intraocular pressure were completely normal. Dilated fundus examination by indirect ophthalmoscopy in the right eye was normal and in the left eye there was a well circumscribed brownish black heavily pigmented small and slightly elevated retinal lesion at the fovea. The tumour mass was located $2.07 \mathrm{~mm}$ temporal to the optic disc [Figure 1]. The maximum horizontal diameter of the lesion was $1.04 \mathrm{~mm}$. The maximum vertical diameter of the lesion was $1.43 \mathrm{~mm}$. There was also premacular gliosis. There was no associated RPE atrophy, macular edema, retinal exudation or subretinal fluid.

Fundus fluorescein angiography (FFA), optical coherence tomography (OCT) and ocular B Scan ultrasonography were performed. Fluorescein angiography showed blockage of fluorescence during all phases of FFA due to pigmented tumour and there was no leakage at fovea [Figure $2 \mathrm{a} \& 2 \mathrm{~b}$ ]. The retinal vessels at macula were minimally dilated and there was no feeder vessels for the tumour mass. The temporal arcades were dragged towards the lesion.

The macular OCT scan was performed using CirrusTM HDOCT Model 4000 (Carl-Zeiss Meditec, Inc, Dublin, California, USA). The High definition OCT image scan - HD 5-line raster scan [Figure 3] of the left eye revealed an abruptly elevated hyper reflective mass with increased optical reflectivity on its inner surface with optical shadowing beneath and well-defined clear cut tumour margins. There was vitreo macular adhesion at the nasal margin of the lesion. The surrounding retina and choroid was normal. The retinal tumour thickness in SD-OCT was 1004 microns $(1 \mathrm{~mm})$. B-scan ocular ultrasonography of the left eye showed a nodular high-echogenic small mass at the macula [Figure 4].

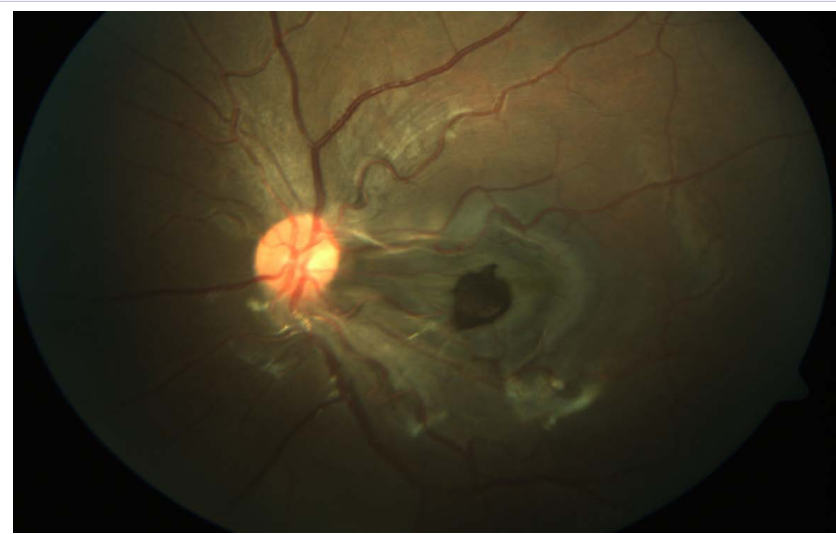

Figure 1: Colour fundus photo of the left eye. Retinal pigment epithelial hamartoma located at the fovea is noted. The arteries of the temporal arcade are dragged towards the lesion with pre-macular gliosis. 
Examination and imaging findings of the right eye were completely normal. In our case, the subfoveal location of the retinal lesion was responsible for the poor visual acuity in the left eye.
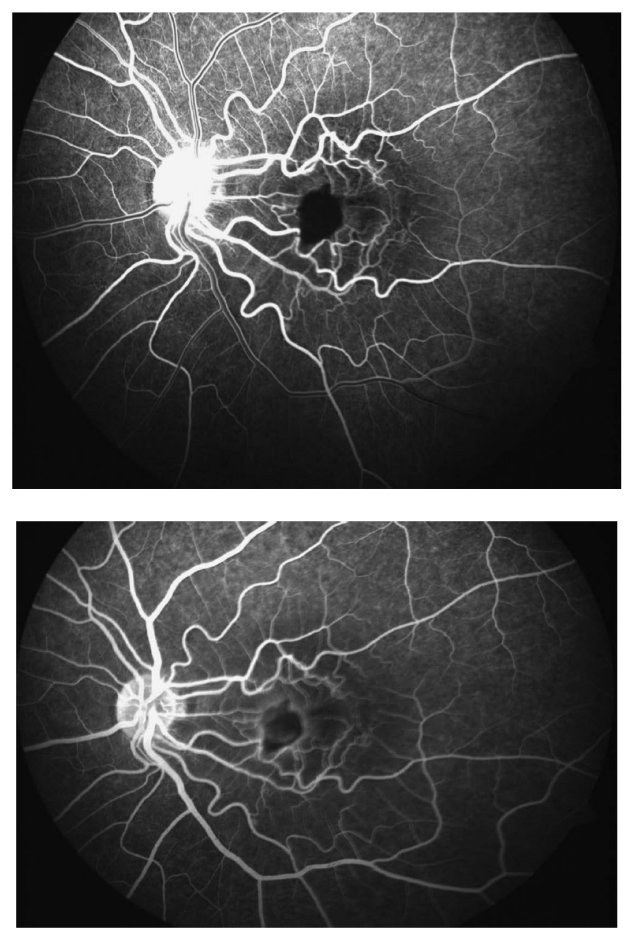

Figure 2: Fundus Fluorescein Angiography of the left eye. Blockage of fluorescence due to pigmented tumour is noted in early phase (2a) and late phase (2b) with no leakage at fovea.

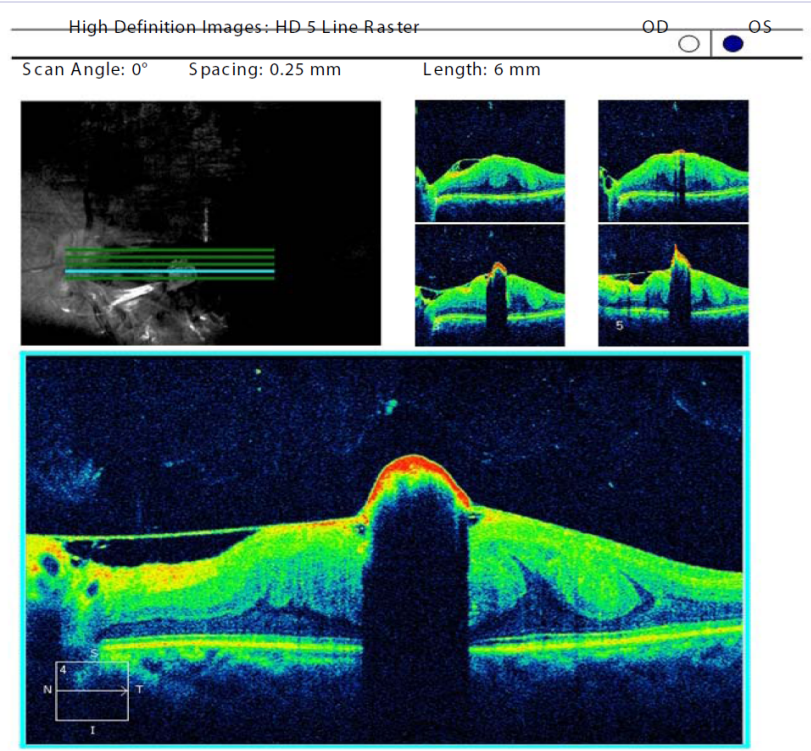

Figure 3: Optical coherence tomography (High Definition) image at the macula of left eye. High surface reflectivity with deep optical shadowing, full thickness retinal involvement with clear cut tumour margins and vitreomacular adhesion at the nasal margin of foveal lesion.

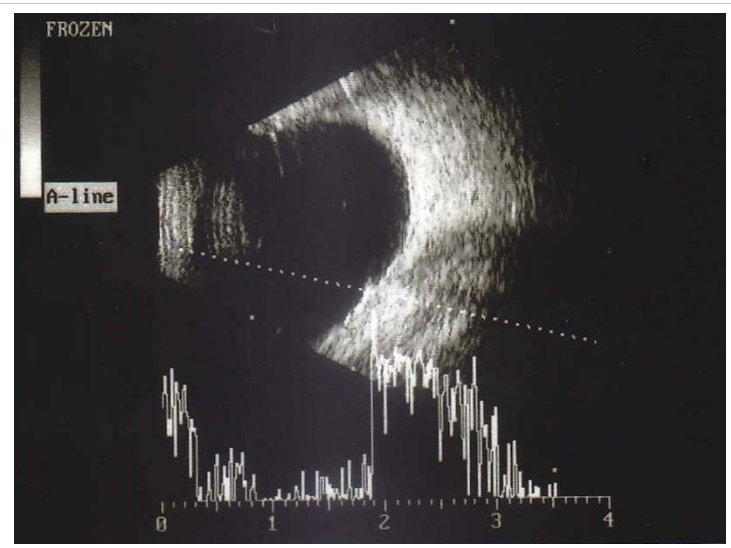

Figure 4: Ocular B-scan ultrasonography (left eye). An echogenic, nodular appearance of the lesion without orbital shadowing is noted.

\section{Discussion}

Tumours and tumour like lesions of the retinal pigment epithelium (RPE) represents a diagnostic problem for the clinician [3]. CSHRPE is incidentally diagnosed in asymptomatic children and young adults [5-6].

Congenital SHRPE was described by Laqua [3] in 1981, based on clinical observations in 2 cases. He reported a 39 year old man with decreased vision who had a jet-black parafoveal tumour of 1 $\mathrm{mm}$ basal dimension that projected through full thickness retina into the vitreous cavity, minimally dilated feeding vessels were noted. This lesion remained stable for 15 years.

The second case was a 15 year old boy with good visual acuity and a $0.5 \mathrm{~mm}$ black full thickness retinal tumour inferior to the foveola. He pointed out that these lesions differed from CSHRPE and other RPE lesions. In 1989, Gass [1] published a review on focal congenital anomalies of the RPE and he coined the term RPE hamartoma. In 2003, Shields [5] documented the largest case series consisting of 5 patients. The OCT findings of CSHRPE were first described by Shields et al. in 2004. Then Shukla et al [4] reported that OCT is a useful, non-invasive tool for the diagnosis of this rare tumour. In 2014, A.C. Barnes et al [8] reported a case of CSHRPE with foveal involvement and secondary epiretinal membrane formation that underwent surgical excision allowing for a detailed histopathological examination of the lesion that showed a nodular proliferation of hyperplastic RPE cells with attached gliotic retina and internal limiting membrane (ILM). The RPE cells showed fibrous metaplasia. CSHRPE is an uncommon presumed congenital lesion that has characteristic ophthalmoscopic, fluroescein angiographic and OCT features. CSHRPE appears ophthalmoscopically as a small localized, elevated black lesion adjacent to fovea. It is usually $<1 \mathrm{~mm}$ in diameter. The lesions generally remains stationary and is not known to grow [2]. The visual prognosis is good especially for lesions located extrafoveally [5]. This lesion involving a full thickness retina may be associated with retinal traction, retinal exudation, dilated feeding and draining retinal vessels and pigmented vitreous cells [5-9]. None of these associated findings was present in our case. 
Fluorescein angiography of CSHRPE showed non fluorescence throughout the angiogram and in the late phase a subtle silhouette of mild hyperfluorescence was noted, lining the temporal edge of the mass that was reported by Shields et al [5] similar findings was seen in our case.

Ocular B-scan ultrasonography demonstrates an echogenic, nodular, small mass in the retina-choroid region with moderate to high internal reflectivity on A scan and without shadowing [4-5]. Similar B scan findings was seen in our case. Cirrus HD Spectral domain OCT macular cube 512 × 128 scan protocol and HD OCT image scan of our case showed an elevated retina at the site of the lesion, increased optical reflectivity on its inner surface, optical shadowing of deeper structures and clearly cut tumour margins.

The surrounding retina and choroid had normal structures. These Oct findings were consistent with those of previously reported cases $[4,7,10,11]$. The normal appearance of the adjacent underlying choriocapillaries and choroid in OCT was considered helpful in excluding the diagnosis of choroidal nevus or melanoma [7]. Thus OCT is quite diagnostic in determining the exact anatomical location of the hyperplastic RPE cells located within the retina having their apex at the vitreo retinal surface. To summarize CSHRPE located extrafoveally usually requires no active treatment since the lesion is non progressive and does not affect visual acuity. While CSHRPE located at fovea results in poor visual acuity and the foveal location of the tumour limits the use of laser photocoagulation or other methods of treatment.

Yet it is important to follow-up these patients over long term to rule out tumour growth and suspicious malignant features. Varied pigmented lesions such as congenital hypertrophy of the RPE, combined hamartoma of the retina and RPE, reactive RPE hyperplasia, choroidal melanoma, choroidal nevus, RPE adenoma and adenocarcinoma of the RPE, intraretinal foreign body and melanocytoma should be considered in the differential diagnosis of CSHRPE [4, 5,7-11]. All these can be ruled out by history, clinical examination, fluorescein angiography and other complementary examination.

\section{References}

1. Gass JD. Focal Congenital anomalies of the retinal pigment epithelium. Eye (Lond). 1989;3(Pt 1):1-18.

2. Shields JA, Shields CL. Tumours and related lesions of the retinal pigment epithelium. In: Shields JA, Shields CL, editors. Atlas of Intra Ocular Tumours. Philadelphia: Lippincot Williams \& Wilkins. 1999:287 - 309 .

3. Laqua H. Tumours and tumour-like lesions of the retinal pigment epithelium. Opthalmologica. 1981;183(1):34-38. doi: 10.1159/000309131.

4. Shukla D, Ambatkar S, Jethani J, Kim R. Optical Coherence tomography in presumed congenital simple hamartoma of the retinal pigment epithelium. Am J Opthalmol. 2005;139(5):945-47. doi: 10.1016/j. ajo.2004.11.037.

5. Shields CL, Sheilds JA, Marr BP, Sperber DE, Gass JD. Congenital simple hamartoma of the retinal pigment epithelium: A study of five cases. Ophthalmology. 2003;110(5):1005 - 1011. doi: 10.1016/S01616420(03)00087-3.

6. Gass JDM. Developmental tumours of the retinal pigment epithelium (RPE) and retina. In: Gass JDM, editor. Stereoscopic atlas of macular diseases, diagnoses and treatment. $4^{\text {th }}$ ed, St. Louis: Mosby-Year Book Inc. 1997:809 - 865.

7. Shields CL, Materin MA, Karatza EC, Shields JA. Optical Coherence tomography of congenital simple hamartoma of the retinal pigment epithelium. Retina. 2004; 24(2):327-328.

8. Barnes AC, Goldman DR, Laver NV and Duker JS. Congenital simple hamartoma of the retinal pigment epithelium: Clinical, optical coherence tomography and histopathological correlation. Eye (Lond). 2014;28(6):765-766. doi: 10.1038/eye.2014.49.

9. L. A. Yannuzzi. "Congenital simple hamartoma of the retinal pigment epithelium," in The Retinal Atlas, L. A. Yanuzzi, Ed., pp. 650-663, Saunders, Beijing, China, 2010.

10. Gotoh M, Yoshikawa H, Kagimoto HTS and Ishibashi T. Congenital simple hamartoma of the retinal pigment epithelium in an Asian. Jpn J Ophthalmol. 2008;52(2):144-145. doi: 10.1007/s10384-008-0509-y.

11. Lopez IM, Guerrero P. Congenital simple hamartoma of the retinal pigment epithelium Optical Coherence Tomography and angiography features. Retina 2006;26(6):704-706. doi: 10.1097/01. iae.0000236504.35268.96. 